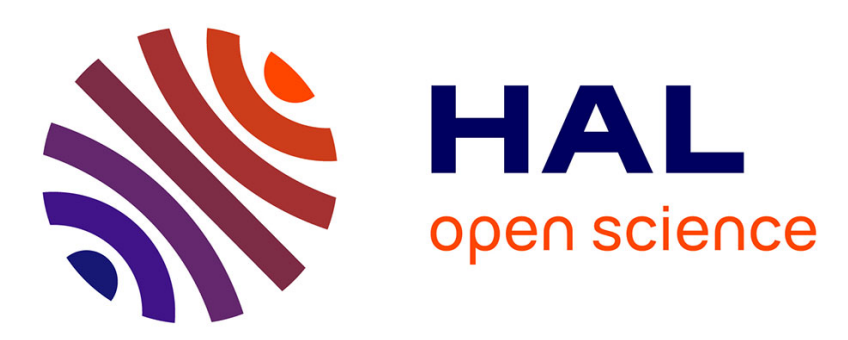

\title{
Application des mesures de susceptibilité magnétique à l'étude de la transition cholestérique $\rightarrow$ nématique
}

\author{
B. Regaya, H. Gasparoux, Jacques Prost
}

\section{To cite this version:}

B. Regaya, H. Gasparoux, Jacques Prost. Application des mesures de susceptibilité magnétique à l'étude de la transition cholestérique $\rightarrow$ nématique. Revue de Physique Appliquée, 1972, 7 (2), pp.8388. 10.1051/rphysap:019720070208300 . jpa-00243609

\section{HAL Id: jpa-00243609 https://hal.science/jpa-00243609}

Submitted on 1 Jan 1972

HAL is a multi-disciplinary open access archive for the deposit and dissemination of scientific research documents, whether they are published or not. The documents may come from teaching and research institutions in France or abroad, or from public or private research centers.
L'archive ouverte pluridisciplinaire HAL, est destinée au dépôt et à la diffusion de documents scientifiques de niveau recherche, publiés ou non, émanant des établissements d'enseignement et de recherche français ou étrangers, des laboratoires publics ou privés. 


\title{
APPLICATION DES MESURES DE SUSCEPTIBILITÉ MAGNÉTIQUE A L'ÉTUde DE LA TRANSITION CHOLESTÉRIQUE $\rightarrow$ NÉMATIQUE
}

\author{
B. REGAYA, H. GASPAROUX et J. PROST \\ Centre de Recherches Paul-Pascal, Domaine Universitaire, 33-Talence
}

(Reçu le 3 janvier 1972, révisé le 23 février 1972)

\begin{abstract}
Résumé. - Une méthode originale d'évaluation du champ de transition correspondant au passage d'une mésophase cholestérique à une mésophase nématique est proposée. L'analyse de la transformation conduit à un bon accord entre nos résultats expérimentaux et la théorie de ce phénomène proposée par De Gennes. A partir de l'évaluation de l'anisotropie magnétique une valeur de la constante d'élasticité $K_{22}$ est déterminée.

Abstract. - An original method to evaluate the transition field strength characteristic of the cholesteric-nematic transition phase is proposed. The transition study shows a good agreement between our experimental results and De Gennes theory. From the knowledge of diamagnetic anisotropy a value of the elastic constant $K_{22}$ is determined.
\end{abstract}

Dans une mésophase cholestérique, les molécules ne se disposent pas exactement parallèles les unes aux autres mais adoptent une configuration hélicoïdale. Si on effectue une coupe de la structure suivant un plan perpendiculaire à l'axe $z$ de la spirale, la disposition des molécules dans le plan est analogue à celle d'un nématique mais la direction privilégiée d'orientation des molécules tourne lentement quand on se déplace suivant l'axe $z$. On obtient ainsi une structure périodique hélicoïdale.

$\mathrm{Si}$ les molécules ont une anisotropie magnétique positive, elles tendent à s'orienter dans la direction du champ magnétique auquel est soumise une mésophase cholestérique. Lorsque la valeur du champ devient supérieure à une valeur limite $H_{\mathrm{c}}$ (champ critique), les molécules deviennent parallèles entre elles dans tout le volume et on obtient une configuration nématique.

Ce phénomène décelé en 1967 par RMN [1] a été interprété indépendamment par de Gennes et R. B. Meyer à partir de la théorie du continuum [2]-[3]. La vérification expérimentale de la théorie de de Gennes a été réalisée ensuite à partir de l'étude de la modification des propriétés optiques d'une mésophase cholestérique sous l'action d'un champ magnétique d'intensité variable [4]-[5].

Le travail présenté ici a pour objet de mettre en évidence la modification de structure à partir de l'évaluation de la susceptibilité magnétique des composés étudiés, tout en essayant de rendre compte de l'allure de la courbe de transition théorique prévue par de Gennes.

I. Principes de la méthode. Conditionnement des échantillons. - Afin de connaître avec la meilleure précision possible la valeur du champ magnétique auquel est soumis le matériau étudié, une prospection soignée de la carte de répartition du champ magnétique dans l'entrefer de l'électroaimant a été effectuée. Le matériau est placé dans une coupelle cylindrique en pyrex de faibles dimensions ( $\phi=4 \mathrm{~mm}, h=4 \mathrm{~mm}$ ) afin que la totalité de l'échantillon soit soumise à des champs magnétiques relativement voisins $\left({ }^{1}\right)$.

En utilisant les abaques donnant la répartition du champ dans l'entrefer nous avons déterminé la position de l'échantillon qui conduit au meilleur compromis possible entre la nécessité d'avoir une force magnétique suffisante (valeur suffisante de $H \partial H / \partial x$ ) et le souci d'éviter que la valeur du champ ne varie trop rapidement au sein du produit, ce qui affecterait la précision de l'évaluation de $H_{\mathrm{c}}\left({ }^{2}\right)$.

Nous avons représenté sur la figure 1 la variation du champ magnétique auquel est soumise la coupelle en fonction de l'intensité du courant et pour différentes positions de la coupelle par rapport à l'axe des pièces polaires.

Nous avons pu définir ainsi des conditions opératoires telles que l'écart maximum, entre la valeur du champ magnétique au centre de la coupelle et à l'une de ses extrémités, n'excède jamais 300 gauss, dans le cas le plus défavorable (correspondant aux champs critiques les plus élevés).

(1) La présence d'un gradient de champ se traduit par une variation de la pression hydıostatique et n'a pas d'effet direct sur l'orientation moléculaire.

(2) On peut remarquer que ce compromis conduit à une évaluation de la susceptibilité magnétique moins précise que celle que nous avions effectuée lorsque la détermination des propriétés magnétiques des produits constituait le seul objectif de l'étude (réf. [6 et 9]). 


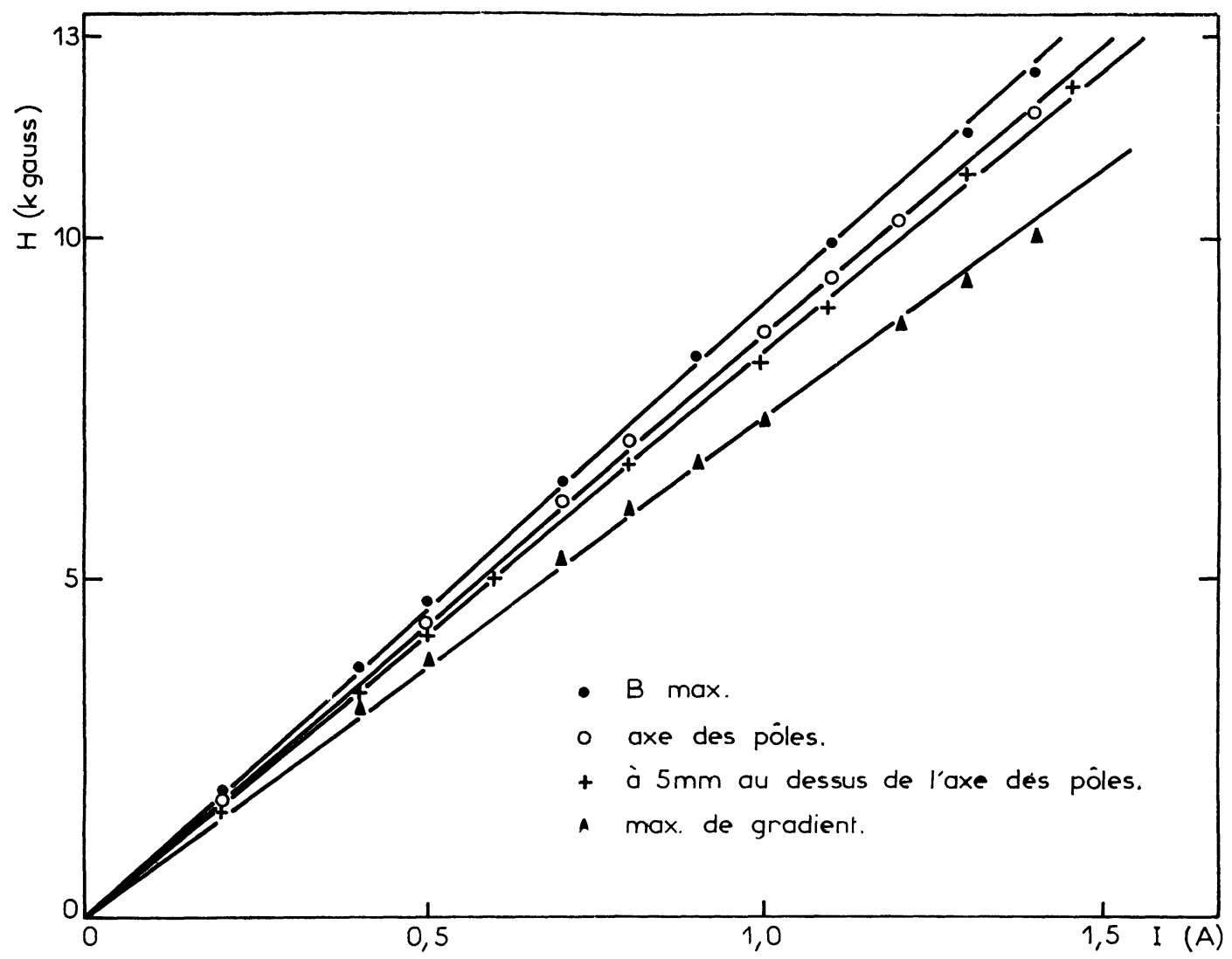

FIG. 1. - Variation de l'induction magnétique en fonction de l'intensité du courant pour différentes positions par rapport à l'axe des pièces polaires.

II. Nature des mésophases cholestériques étudiées. - Nous avons étudié des échantillons constitués d'un mélange de (méthoxybenzilidène butyl aniline) ou MBBA de structure nématique et de carbonate-2(2 Ethoxy éthoxy) éthyl de cholestérol de structure cholestérique.

Des travaux antérieurs ont montré que le mélange ainsi obtenu était de structure cholestérique.

Quatre mélanges ont été étudiés correspondant respectivement à des concentrations en ester cholestérique égales à 0,$47 ; 0,76 ; 0,86$ et $1 \%$.

III. Variation de la susceptibilité magnétique d'un mélange cholestérique en fonction de l'intensité du champ magnétique. - A) RÉSULTATS EXPÉRIMENTAUX. - Les mesures ont été effectuées à l'aide d'une balance magnétique de grande précision suivant la méthode de Faraday [6].

Nous avons reporté sur la figure 2, les résultats relatifs aux quatre produits définis précédemment. Nous constatons qu'à partir d'une certaine valeur du champ magnétique, la susceptibilité mesurée totalement déroulée et nous avons pu ainsi mesurer reste constante. La spirale cholestérique est alors

le champ critique $H_{\mathrm{c}}$ correspondant à chacun des échantillons.

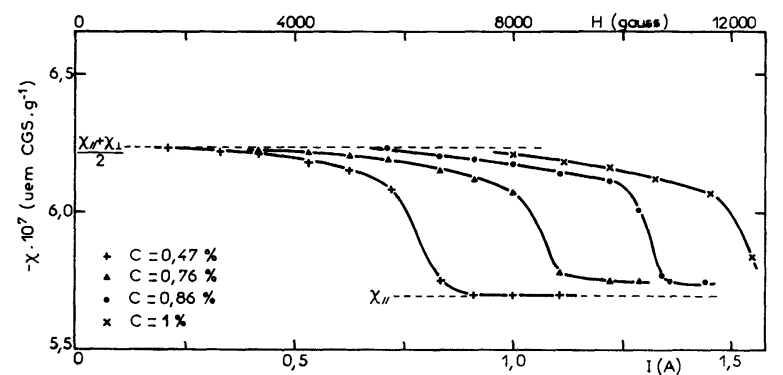

FIg. 2. - Variation de la susceptibilité magnétique $\chi$ en fonction du champ pour les différents échantillons.

B) COMPARAison de nOS RÉSUltatS AVEC LA PRÉVISION THÉORIQUE DE DE GENNES. - 1) Théorie de la transition. - a) Rappel de la théorie de de Gennes. - A chaque point matériel $M(x, y, z)$, est associé un vecteur directeur n (Fig. 3), représentant la direction de l'axe optique de la phase mésomorphe, localement uniaxe. En admettant, pour le vecteur directeur, une expression de l'énergie élastique de type

$$
0,76 \%
$$$$
0,86 \%
$$$$
1 \%
$$

Concentration en ester
des échantillons (en poids)

$H_{\mathrm{c}}$ (gauss)

$6650 \pm 50$
$8850+50$ 


\section{APPLICATION DES MESURES DE SUSCEPTIBILITÉ MAGNÉTIQUE}

Hooke, et en tenant compte des conditions de symétrie, on arrive à l'expression suivante de l'énergie libre par unité de volume d'un cristal cholestérique [7]-[8].

$$
\begin{aligned}
F=\frac{1}{2}\left[K_{11}(\operatorname{div} \mathbf{n})^{2}+\right. & K_{22}\left(\mathbf{n} \cdot \operatorname{rot} n+q_{0}\right)^{2}+ \\
& \left.+K_{33}[(\mathbf{n} \cdot \nabla) \mathbf{n}]^{2}\right]-\frac{1}{2} \mathbf{H} \cdot \chi \cdot H .
\end{aligned}
$$

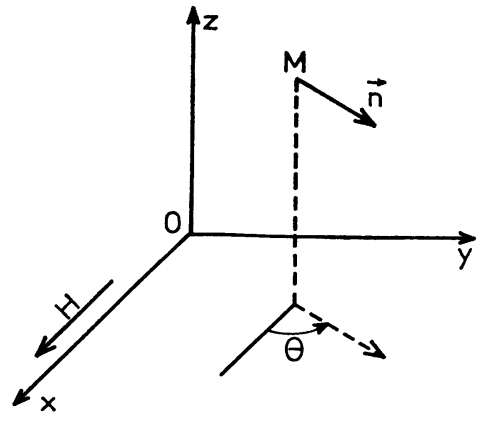

FIG. 3.

$K_{11}, K_{22}, K_{33}$ sont respectivement les constantes élastiques, de déformation en gerbe, de torsion et de flexion. $q_{0}$ est le vecteur d'onde de l'hélice, c'est-à-dire que si $p_{0}$ est la période en champ nul :

$$
p_{0}=\frac{\pi}{q_{0}}\left({ }^{3}\right)
$$

La structure correspondant à l'équilibre est celle qui minimise l'énergie libre, on obtient une solution périodique de période $p$, telle que :

$$
p=p_{0}\left(\frac{2}{\pi}\right)^{2} K(k) E(k)
$$

$K(k)=F(k, \pi / 2)$ intégrale elliptique de première espèce.

$E(k)=E(k, \pi / 2)$ intégrale elliptique de seconde espèce.

La constante $k$ est déterminée par la condition de minimisation de $F$ :

$$
\frac{H}{q_{0}} \sqrt{\frac{\Delta \chi}{K_{22}}}=\frac{\pi}{2} \frac{k}{E(k)} .
$$

Pour $k=1$, l'intégrale elliptique de première espèce diverge, le pas de l'hélice tend vers l'infini, le composé est alors "nématique ». Ceci se produit pour une valeur du champ donnée par (2) :

$$
H_{\mathrm{c}}=\frac{\pi}{2} \sqrt{\frac{K_{22}}{\Delta \chi}} q_{0}=\frac{\pi^{2}}{2} \sqrt{\frac{K_{22}}{\Delta \chi}} \cdot \frac{1}{p_{0}} .
$$

La détermination du champ critique permet donc de déterminer le rapport $K_{22} / \Delta \chi$, si l'on connaît le pas initial de la structure.

(3) La relation est bien $p_{0}=\pi / q_{0}$ et non $p_{0}=2 \pi / q_{0}$, car les orientations - d et $+\mathbf{d}$ sont physiquement identiques (pas d'effet ferro-électrique spontané dans les cristaux liquides). b) Variation de la susceptibilité mesurée en fonction du champ magnétique appliqué. - Remarquons que le calcul suivant s'applique à tout tenseur de rang deux. La valeur de la susceptibilité mesurée est :

$$
\chi=\frac{1}{V} \int_{V} \chi_{x x} \mathrm{~d} v
$$

Compte tenu de l'homogénéité de la structure dans le plan $x y$, et pour des pas petits devant les dimensions de la cellule :

$$
\chi=\frac{1}{p} \int_{0}^{p}\left(\chi_{\|} \cos ^{2} \theta+\chi_{\perp} \sin ^{2} \theta\right) \mathrm{d} z\left({ }^{4}\right) .
$$

Les axes sont choisis de telle sorte que $O z$ coïncide avec l'axe de l'hélice. Posant $\Psi=(\pi / 2)-\theta$, on obtient :

$$
\chi=\frac{1}{p} \int_{-\pi / 2}^{+\pi / 2}\left(\chi_{\|}+\cos ^{2} \Psi\left(\chi_{\perp}-\chi_{\|}\right)\right)\left(\frac{\partial z}{\partial \Psi}\right) \mathrm{d} \Psi .
$$

Avec $h=H / H_{\mathrm{c}} \cdot \pi / 2$, l'expression donnant la valeur de la susceptibilité prend la forme :

$$
\begin{array}{r}
\chi=\chi_{\|}+\left(\chi_{\perp}-\chi_{\|}\right) \frac{\pi}{2 k h}\left(\frac{k^{2}-1}{E(k)}+\frac{1}{K(k)}\right)= \\
=\chi_{\|}+\left(\chi_{\perp}-\chi_{\|}\right) A .
\end{array}
$$

$\alpha)$ Pour $h$ faible $(k \ll 1)$, nous pouvons faire un développement limité des fonctions elliptiques suivant les puissances de $k$.

Ce qui donne pour $E(k)$ et $K(k)$ :

$$
\begin{aligned}
& E(k)=\frac{\pi}{2}\left[1-\frac{k^{2}}{4}-\frac{3}{64} k^{4}+0\left(k^{6}\right)\right] \\
& K(k)=\frac{\pi}{2}\left|1+\frac{k^{2}}{4}+\frac{9}{64} k^{4}+0\left(k^{6}\right)\right| .
\end{aligned}
$$

Soit encore :

$$
\frac{k^{2}-1}{E(k)}+\frac{1}{K(k)}=\frac{2}{\pi}\left(\frac{k^{2}}{2}\right)
$$

en limitant le développement à l'ordre 2 .

Comme d'autre part, l'équation 2 peut s'écrire :

$$
h=\frac{\pi}{2} \frac{k}{E(k)} \text {. }
$$

Soit :

$$
h=k+0\left(k^{2}\right) \text {. }
$$

(4) $\chi_{\|}$représente la susceptibilité magnétique dans une direction parallèle à un vecteur directeur $\mathbf{n}$ qui donne en chaque point la direction d'alignement des molécules.

$\chi_{\perp}$ représente la susceptibilité magnétique suivant une direction perpendiculaire à $\mathbf{n}$. 
Et finalement :

$$
\chi=\frac{\chi \|+\chi_{\perp}}{2}+0^{\prime}\left(k^{2}\right)
$$

$0\left(k^{2}\right)$ et $0^{\prime}\left(k^{2}\right)$ représentant des fonctions tendant vers zéro plus vite que $k^{2}$.

$\beta$ ) A la transition, on a : $h=\pi / 2$ et $k=1$.

D'où : $\chi=\chi_{\|}$.

Le résultat est donc celui obtenu avec un nématique classique.

y) Pour des champs intermédiaires, on détermine $k$ par l'éq. (4) et le reporte dans l'éq. (3). On obtient alors une courbe que l'on peut représenter en coordonnées réduites et qui peut être confrontée avec l'expérience. (Courbe théorique de la figure 4.)

2) Comparaison de nos résultats avec la théorie. a) Variation de la susceptibilité magnétique en fonction du champ. - Nous avons tout d'abord vérifié que dans les cas limites $\left(H \ll H_{\text {c }}\right.$ et $\left.H>H_{\mathrm{c}}\right)$ les valeurs expérimentales de $\chi$ correspondaient aux grandeurs calculées en prenant pour valeurs de $\chi_{\|}$ et $\chi_{\perp}$ les grandeurs caractérisant le MBBA à $26^{\circ} \mathrm{C}$ et que nous avons déjà publiées par ailleurs [9]. Le tableau suivant montre la bonne concordance entre ces valeurs.

$$
\begin{array}{ccc}
\chi \chi & -\chi_{\text {exp }} \times 10^{7} & -\chi_{\text {cal }} \times 10^{7} \\
H \ll H_{\mathrm{c}} & 6,22 & \chi_{\text {cal }}=\frac{\chi_{\|}+\chi_{\perp}}{2}=6,29 \\
H>H_{\mathrm{c}} & 5,70 & \chi_{\text {cal }}=\chi_{\|}=5,80
\end{array}
$$

A partir des valeurs des susceptibilités magnétiques obtenues et des intensités des champs magnétiques de transition, nous avons calculé les valeurs de :

$$
X_{i}=\left(\frac{\pi}{2} \frac{H_{i}}{H_{\mathrm{c}}}\right)=h_{i} \quad \text { et } \quad Y_{i}=\frac{1}{2} \frac{\chi_{i}-\chi_{\mathrm{Nem}}}{\chi_{\max }-\chi_{\mathrm{Nem}}}
$$

pour chaque point $i$ de chaque courbe, afin de vérifier que la courbe $Y_{i}=f\left(h_{i}\right)$ correspond bien à la courbe $A=f\left(h_{i}\right)$.

$\chi_{i}$ : susceptibilité magnétique du point $i$ étudié, $\chi_{\mathrm{Nem}}$ : susceptibilité magnétique du mélange nématique (après transition),

$\chi_{\max }$ : susceptibilité magnétique du mélange cholestérique,

$H_{i}$ : champ magnétique correspondant au point $i$ étudié,

$H_{\mathrm{c}}$ : champ magnétique critique de transition pour l'échantillon étudié.

Nous avons porté les valeurs $X_{i}$ en abscisses et $Y_{i}$ en ordonnées et ceci pour les quatre échantillons (Fig. 4) ; nous remarquons que ces différents points se placent bien sur la courbe théorique obtenue à partir des calculs précédents.

On peut donc dire que l'étude des propriétés magnétiques est un moyen commode d'analyser les transitions cholestérique $\rightarrow$ nématique. La détermination des champs critiques correspondants peut en outre être évaluée avec une assez bonne précision à l'aide du procédé que nous venons de décrire ici.

c) Vérification de la détermination de $H_{\mathrm{c}}$; Application à la mesure de $K_{22}$. - Le mélange d'une mésophase cholestérique et d'une mésophase nématique

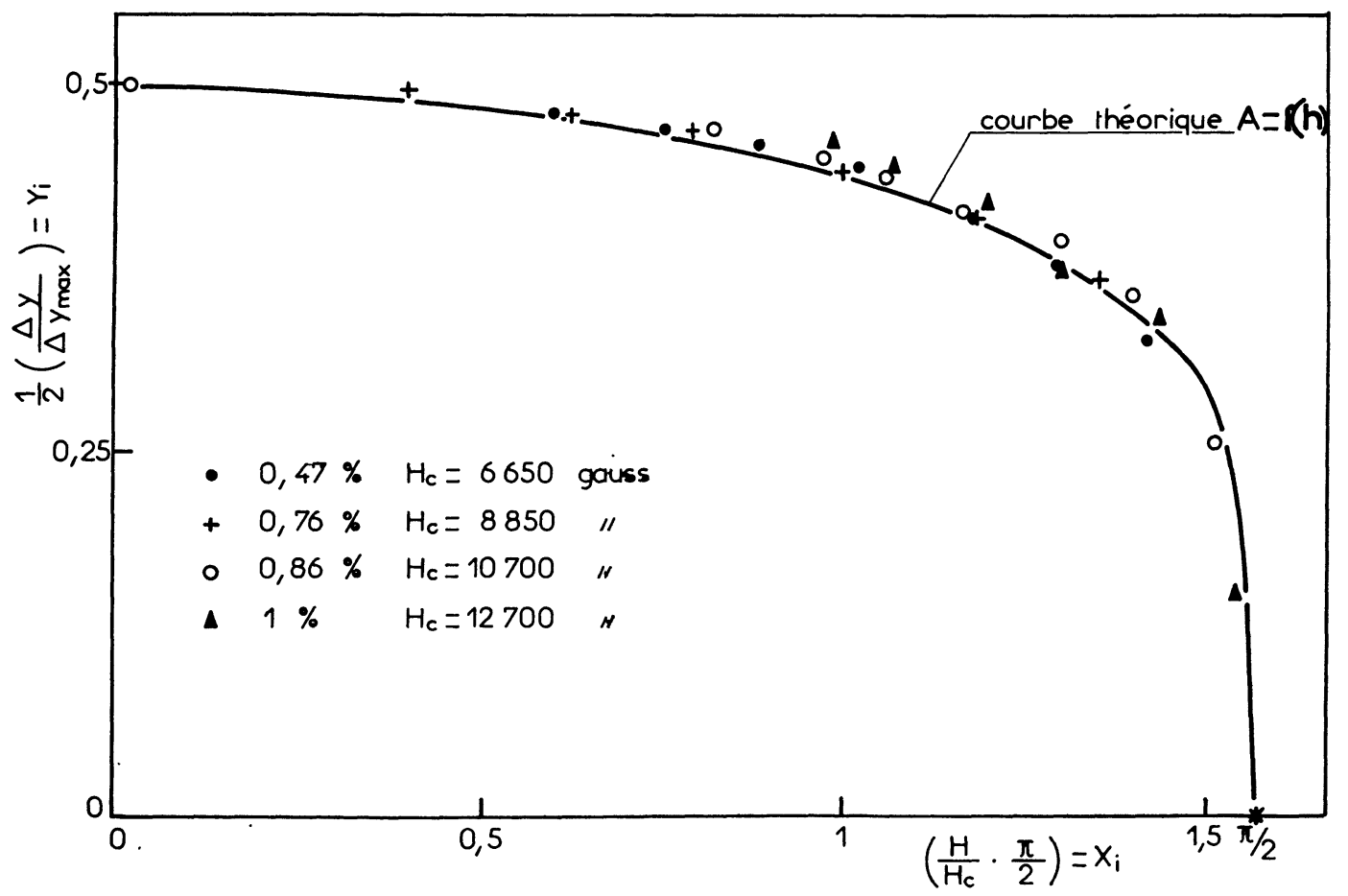

FIG. 4 . - Courbe en coordonnées réduites permettant de comparer nos résultats expérimentaux à la théorie de de Gennes. 
est, comme nous l'avons déjà remarqué, cholestérique. Le pas de la structure obtenue étant évidemment fonction de la concentration en produit optiquement actif. On peut faire le développement suivant :

$$
\frac{1}{p_{0}}=\alpha c+\varepsilon c^{2}
$$

$c:$ concentration en espèce optiquement active.

Le domaine de variation linéaire est assez grand $(c<0,1)$ [4], [10], [11] et peut être justifié théoriquement [12].

Si $\quad c=0 \rightarrow \frac{1}{p_{0}}=0 \quad$ nématique pur.

Nous avons vu, d'autre part, que la variation du champ critique avec cette période obéissait à la loi :

$$
H_{\mathrm{c}}=\frac{\pi^{2}}{2} \sqrt{\frac{K_{22}}{\Delta \chi}} \frac{1}{p_{0}} .
$$

Soit encore :

$$
H_{\mathrm{c}}=\frac{\pi^{2}}{2} \sqrt{\frac{K_{22}}{\Delta \chi}} \alpha c
$$

Nous avons porté sur la figure 5 les valeurs des champs critiques que nous avons obtenues en fonction des concentrations correspondantes $c_{i}$. On peut constater que la variation linéaire de $H_{\mathrm{c}}$ en fonction de $\mathrm{c}$ est bien vérifiée. Ceci confirme les résultats déjà obtenus par voie optique (4) et constitue a fortiori une bonne confirmation de la validité de la méthode d'évaluation de $H_{\mathrm{c}}$ proposée ici.

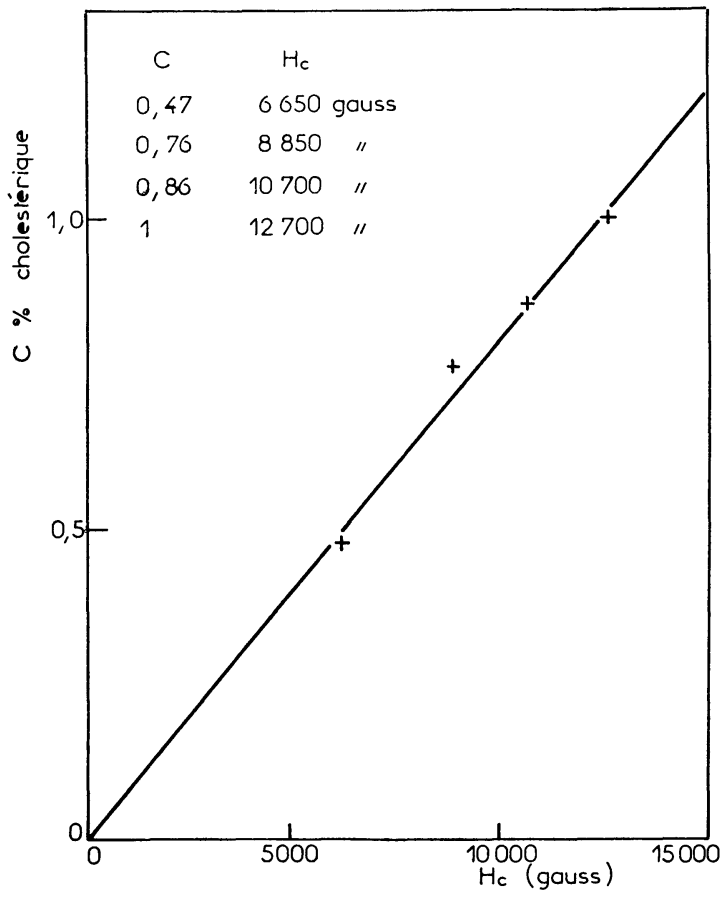

FIG. 5. - Variation du champ critique en fonction de la concentration.
Afin de compléter cette étude, nous avons déterminé $p_{0}$ pour chaque concentration. Nous avons utilisé, pour cela, deux méthodes :

a) Une première inspirée par les travaux de Cano utilise un " coin de mésophase ".

La distance $\left(n .2 p_{0}\right)$ séparant $n$ lignes de disinclinaison est mesurée simplement de la manière suivante : nous réalisons l'image du " coin de mésophase " sur un écran et repérons la position d'un nombre $k_{1}$ de lignes de disinclinaison.

Nous appliquons ensuite un champ magnétique $H>H_{\mathrm{c}}$, et comptons le nombre $k_{1}^{\prime}$ de franges d'interférence (lumière polarisée à $\pi / 4$ de l'axe optique) séparant les deux repères.

Nous pouvons alors écrire :

$$
2 k_{1} p_{0}=\frac{k_{1}^{\prime} \lambda}{\Delta n}
$$

Nous avons utilisé un laser hélium néon $(\lambda=6328 \AA)$ et pris pour valeur de $\Delta n=0,205$ à $T=25^{\circ} \mathrm{C}$ [13].

b) Nous avons aussi utilisé la texture planaire de Grandjean, visible sur une goutte libre, la mesure de la distance séparant deux plans étant semblable à celle décrite précédemment. Dans ce cas :

$$
k_{2} p_{0}=\frac{k_{2}^{\prime} \lambda}{\Delta n} \text {. }
$$

L'identité des résultats obtenus par les deux méthodes apporte une confirmation du fait que les lignes de Cano sont des lignes doubles [14].

Connaissant $p_{0}$ pour chaque concentration, on peut alors tracer la courbe $H_{\mathrm{c}}=f\left(1 / p_{0}\right)$. La droite obtenue (Fig. 6) confirme la bonne cohérence de l'ensemble des résultats.

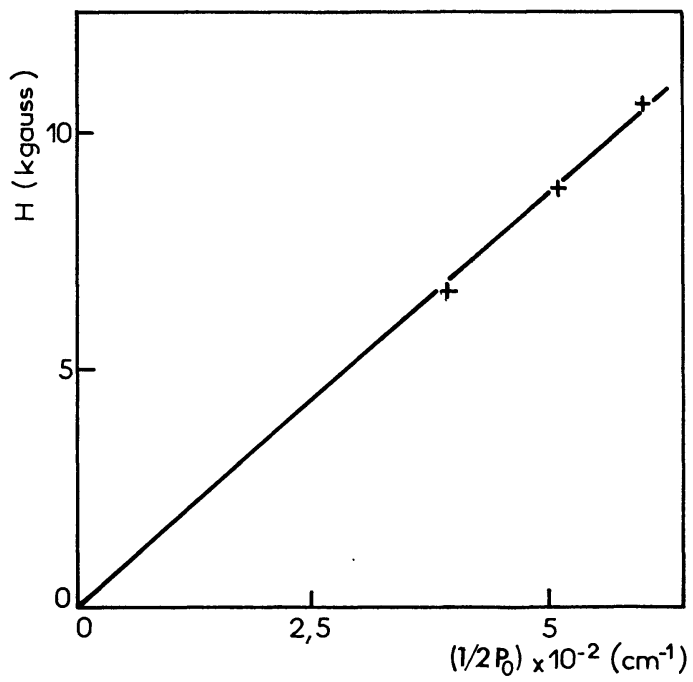

Fig. 6. - Variation du champ critique en fonction de la période $p_{0}$ de la structure hélicoïdale.

La pente de cette droite est égale à

$$
\frac{\pi^{2}}{2} \sqrt{\frac{K_{22}}{\Delta \chi}} \text {. }
$$


Il est donc possible, connaissant $\Delta \chi$ que nous avons mesuré précédemment, d'évaluer la constante de torsion $K_{22}$ par un moyen différent de ceux utilisés jusqu'à présent.

La valeur obtenue pour $\frac{K_{22}}{\Delta \chi}\left(\frac{K_{22}}{\Delta \chi}=3,1\right)$ est en bon accord avec les déterminations faites par d'autres auteurs $\left({ }^{6}\right)$; elle nous permet de proposer comme valeur du coefficient d'élasticité relatif à la torsion : $K_{22} \simeq 3,5 \times 10^{-7}$ uem CGS (à $25^{\circ} \mathrm{C}$ ).

Conclusion. - On peut retenir, en conclusion, les points suivants :

(๑) Colloque sur les cristaux liquides (Pont-à-Mousson, 1971).
- L'analyse des résultats présentés ici montre que l'apport des mesures magnétiques permet d'évaluer la constante de torsion $K_{22}$ d'un matériau mésomorphe.

- La bonne concordance entre l'exploitation des résultats expérimentaux et la théorie de de Gennes est la preuve qu'une étude complète des propriétés magnétiques des substances cholestériques est maintenant possible.

On peut, en effet, affirmer que lorsque $H<H_{\mathrm{c}}$, la grandeur expérimentale correspond à la valeur $\frac{\chi_{\perp}+\chi_{\|}}{2}$ si l'anisotropie moléculaire est positive.

\section{Bibliographie}

[1] Sackmann (H.), Synder (L. C.), Meiboom (S.), S. Amer. Chim. Soc., 1967, 89, 5981.

[2] Meyer (R. B.), Appl. Phys. Letters, 1968, 12, 281.

[3] de Gennes (P. G.), Sol. Stat. Comm., 1968, 163.

[4] Durand (G.), Léger (L.), Rondelez (F.) and VeysSIÉ (M.), Phys. Rev. Letters, 1969, 22, 227.

[5] Meyer (R. B.), Appl. Phys. Letters, 1969, 14, 208.

[6] Regaya (B.) et Gasparoux (H.), C. R. Acad. Sci. Paris, 1971, 272, 724.

[7] Frank (F. C.), Disc. Faraday, Soc., 1958, 25, 1.
[8] De Gennes (P. G.), Proceedings of the $2^{\text {nd }}$ Intern Liquid Crystal Conference KENT (1968).

[9] Gasparoux (H.), Regaya (B.) et Prost (J.), $C$. $R$. Acad. Sci. Paris, 1971, 272, 1168.

[10] Cano (R.), C. R. Acad. Sci. Paris, 1960, 251, 1139.

[11] NaKagiri (T.), Phys. Letters, 1971, A, 36A, 427.

[12] Goossens, Mol. Cryst. and Liq. Crystals, 1971, 12, 237.

[13] Brunet-Germain (M.), C. R. Acad. Sci. Paris, 1970, 271 , série $B, 1075$.

[14] Groupe expérimental d'étude des cristaux liquides d'Orsay, J. Physique, 1969, 30, C 438. 